\title{
Improving the precision of linear optics measurements based on turn-by-turn beam position monitor data after a pulsed excitation in lepton storage rings
}

\author{
L. Malina* \\ CERN, Geneva 23, Switzerland and University of Oslo, 0316 Oslo, Norway \\ J. Coello de Portugal, T. Persson, P. K. Skowroński, and R. Tomás \\ CERN, Geneva 23, Switzerland
}

A. Franchi and S. Liuzzo

ESRF, CS 40220, 38043 Grenoble CEDEX 9, France

(Received 24 March 2017; published 4 August 2017)

\begin{abstract}
Beam optics control is of critical importance for machine performance and protection. Nowadays, turnby-turn (TbT) beam position monitor (BPM) data are increasingly exploited as they allow for fast and simultaneous measurement of various optics quantities. Nevertheless, so far the best documented uncertainty of measured $\beta$-functions is of about $10 \%$ rms. In this paper we compare the $\beta$-functions of the ESRF storage ring measured from two different TbT techniques- the N-BPM and the Amplitude methods - with the ones inferred from a measurement of the orbit response matrix (ORM). We show how to improve the precision of TbT techniques by refining the Fourier transform of TbT data with properly chosen excitation amplitude. The precision of the N-BPM method is further improved by refining the phase advance measurement. This represents a step forward compared to standard TbT measurements. First experimental results showing the precision of $\beta$-functions pushed down to $4 \%$ o both in TbT and ORM techniques are reported and commented.
\end{abstract}

DOI: 10.1103/PhysRevAccelBeams.20.082802

\section{INTRODUCTION}

Performance of today's accelerators puts stringent requirements on beam optics control. Moreover, the time spent on optics measurements should be minimized to maximize machine availability. Traditionally, beam optics measurement methods based on the beam closed orbit have been used in synchrotron light sources [1], but the time needed for the measurement could reach several tens of minutes. On the other hand, analysis of turn-by-turn ( $\mathrm{TbT}$ ) beam position monitor (BPM) data allows for simultaneous measurement and correction of various optics quantities as demonstrated in [2-6]. Several beam optics correction methods were compared in [7-9], with typical relative error in the measured $\beta$-functions of about $10 \%$ rms. In machines requiring a $\beta$-beating within $10 \%$ [10], it is then necessary to devise optics measurements with better accuracy and precision.

A general analytical study of errors of TbT techniques is reported in [11]. Different linear optics measurement methods applied to the ESRF storage ring were already

\footnotetext{
*lukas.malina@cern.ch

Published by the American Physical Society under the terms of the Creative Commons Attribution 4.0 International license. Further distribution of this work must maintain attribution to the author(s) and the published article's title, journal citation, and DOI.
}

compared in [12]. Both works revealed that the key points for a better accuracy are the dependence on the lattice models, as well as by the amplitude of the betatron oscillation vs the BPM resolution and the lattice nonlinearities. In machines with strong nonlinear magnetic elements, such as third-generation and future light sources, the linear analysis of TbT data can be biased by nonlinear terms if the beam excitation is too large. In the case of the ESRF storage ring, it is found that amplitudes greater than $1 \mathrm{~mm}$ at $\beta_{x}=38 \mathrm{~m}$ (action variable, $J_{x}=1.3 \times 10^{-8} \mathrm{~m}$ ) are already affected by such terms. In principle lower excitations would be possible by firing the kicker magnet with a weaker current, though the one used at the ESRF storage ring suffers from stability and reproducibility issues at low pulse currents.

We applied two beam optics measurement techniques to TbT data from the ESRF storage ring: the N-BPM method [13] and the Amplitude approach [14]. Both procedures are based on the harmonic analysis of TbT data from all BPMs. The beam is excited in both planes by pulsed magnets (kickers inducing free oscillations). The Amplitude technique evaluates the $\beta$-function from the amplitude of the tune spectral line at each BPM and is biased by BPM calibration errors as discussed for example in [15]. The $\mathrm{N}$-BPM method extracts the $\beta$-function from the BPM phase advance computed from the phase of the tune line as described in [13]. The $\beta$-functions measured with these two 
techniques are compared to those inferred from the measurement and fit of the orbit response matrix (ORM), i.e., of the closed orbit response to a unit change of orbit corrector strengths [16]. The machine model is fitted to reproduce the measured matrix, namely dipole and quadrupole tilts, dipole and quadrupole strength errors, as well as BPM calibration factors. This fit provides an effective model (called the ORM model). The ORM measurement routinely carried out at the ESRF takes about 20 minutes. More generally, the measurement time scales with the length of the storage ring, whereas the one of the $\mathrm{TbT}$ techniques is a matter of at most seconds. However, the switch between $\mathrm{TbT}$ and orbit modes may require up to twenty minutes, as the ESRF storage ring is equipped with BPM electronics [17], which are not suitable for quick switch between the modes.

In lepton machines synchrotron radiation results in the damping of the $\mathrm{TbT}$ transverse motion after a pulsed excitation influencing the analysis of TbT data in two main aspects. First, the evaluation of BPM phase advance, from the phase of the tune spectral line, shows a dependence on the choice of the first turn of the TbT batch of data to be analyzed (even at the level of single turns). A correction to this issue was found in [18]. Second, the natural reduction of the oscillation amplitude over time can be used to obtain a batch of TbT data for the harmonic analysis a few milliseconds after the kicker pulse (strong enough to ensure its stability) with an amplitude sufficiently low to prevent non-linear terms from spoiling the linear analysis of the tune line.

Here we define the terms of: accuracy - the difference to the true value and precision-the spread of the measurements including the known systematic effects. Assuming we have the quantity which has a true value of $1.00,1.00 \pm$ 0.01 is precise and accurate, $0.90 \pm 0.01$ is precise, but inaccurate and $1.0 \pm 0.1$ is imprecise but accurate estimate of the value.

The paper is structured as follows. In Sec. II the measurement setup is outlined, whereas an error analysis of the different measurement techniques based on singleparticle simulations of the ESRF storage ring is detailed in Sec. III. Experimental results are eventually discussed and compared in Sec. IV.

\section{DEDICATED OPTICS MEASUREMENT}

A special sextupole setting was designed and implemented to have both linear chromaticity and amplitude detuning close to zero, resulting in at least several hundred turns of exploitable data for the harmonic analysis.

Optics measurements were performed with transverse oscillation decoherence times of about 740 and 1850 turns in the horizontal and vertical planes, respectively. The synchrotron radiation damping time is about 2500 turns. The fractional betatron tunes were close to nominal values at 0.4414 in the horizontal plane and 0.3899 in the vertical plane. The measured linear chromaticity was -1.0 and +0.2 in the two planes (compared to the operational values of 8 and 13, respectively). 330 bunches with a total current of $7 \mathrm{~mA}$ were stored to avoid beam instabilities and position interlocks during the beam excitation. Prior to TbT measurements, linear lattice errors $(\beta$-beating, dispersion and coupling) were measured and corrected via ORM analysis reaching typical operational values, i.e. $\sim 5 \%$ and $\sim 3 \% \mathrm{rms}$ $\beta$-beating in the two transverse planes and an emittance ratio of $\epsilon_{y} / \epsilon_{x} \simeq 1 \%$. A second ORM was measured and fit to evaluate the $\beta$-functions to be used as reference. After that, BPMs were switched to TbT mode and data with maximum amplitude in the range from $0.7 \mathrm{~mm}$ $\left(J_{0}=6.5 \times 10^{-9} \mathrm{~m}\right)$ to $1.4 \mathrm{~mm}\left(J_{0}=2.6 \times 10^{-8} \mathrm{~m}\right)$ at $\beta=38 \mathrm{~m}$ were acquired. The beam was excited by kickers located at $\beta_{x}=5.1 \mathrm{~m}$ and $\beta_{y}=30.5 \mathrm{~m}$. The time difference between the ORM and TbT measurements was about 3 hours. TbT measurements were repeated for 5 different beam energies (with a $\pm 0.168 \%$ span of $\Delta p / p$ ). For each kicker and energy setting, ten acquisitions were taken.

\section{PRECISION AND ERROR ANALYSIS}

The performance of a harmonic analysis depends, among other elements, on the number of analyzed turns. Simulations have been carried out in order to evaluate the best number of turns and kicker strength ensuring the highest measurement precision. Single particles with various initial transverse displacements were tracked along the lattice of the ESRF storage ring. The lattice $\beta$-functions are shown in Fig. 1. The average horizontal and vertical $\beta$-functions are comparable.

BPM noise in TbT mode is estimated from singular value decomposition (SVD)-cleaning [19-21] of measured data. The decomposed TbT data are recomposed using only the 12 largest singular values, this way the noise floor is removed and the data are cleaned. The rms of the
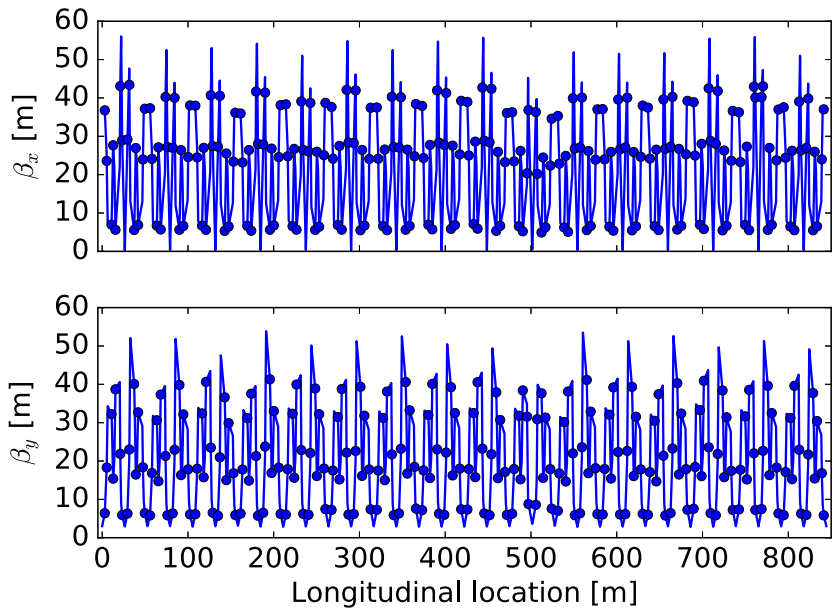

FIG. 1. The lattice $\beta$-functions along the ESRF storage ring. The markers refer to BPM positions. 
differences between the raw and cleaned data for each BPM provided an average noise of $9.6 \mu \mathrm{m}$ in the horizontal plane and $8.8 \mu \mathrm{m}$ in the vertical plane. This BPM noise was added to the simulated TbT data before being analysed using different numbers of turns with the initial displacement of $0.55 \mathrm{~mm}$ (horizontal) and $0.15 \mathrm{~mm}$ (vertical) at $\beta_{x}=38 \mathrm{~m}$ and $\beta_{y}=2.9 \mathrm{~m}$ (initial actions: $J_{x 0}=4.0 \times 10^{-9} \mathrm{~m}$ and $\left.J_{y 0}=3.9 \times 10^{-9} \mathrm{~m}\right)$. Note that the actions of the horizontal and vertical kicks are very similar. The errors of the retrieved BPM phase advance and $\beta$-beating as a function of the number of turns are shown in Figs. 2 and 3, respectively. Because the errors disappeared by removing the sextupoles in the simulation it can be concluded that they are dominated by lattice nonlinearities.

As expected from [22], the degradation of the accuracy in evaluation of phase advance and $\beta$-function below 1200 turns (blue curves in Figs. 2 and 3 ) is largely removed when the tune averaged over all BPMs is used to define the tune spectral line (red curves), instead of using the tune peak detected at each single BPM. Therefore in the analysis of experimental data the tune line is forced to be equal to the average tune.

Larger transverse beam excitations modify the content of the tune spectral line, whose amplitude and phase include non-linear terms (proportional to the initial amplitude) stemming from sextupoles in the case of ESRF storage ring $[4,11]$. Figure 4 shows the artificial $\beta$-beating, inferred from the N-BPM method, applied to simulated data with different initial displacements. Up to $45 \%$ o peak artificial $\beta$-beating is observed with an initial amplitude of $4.4 \mathrm{~mm}$ at $\beta_{x}=38 \mathrm{~m}$ $\left(J_{x 0}=2.5 \times 10^{-7} \mathrm{~m}\right)$. To decrease the inaccuracy to $2 \%$ o
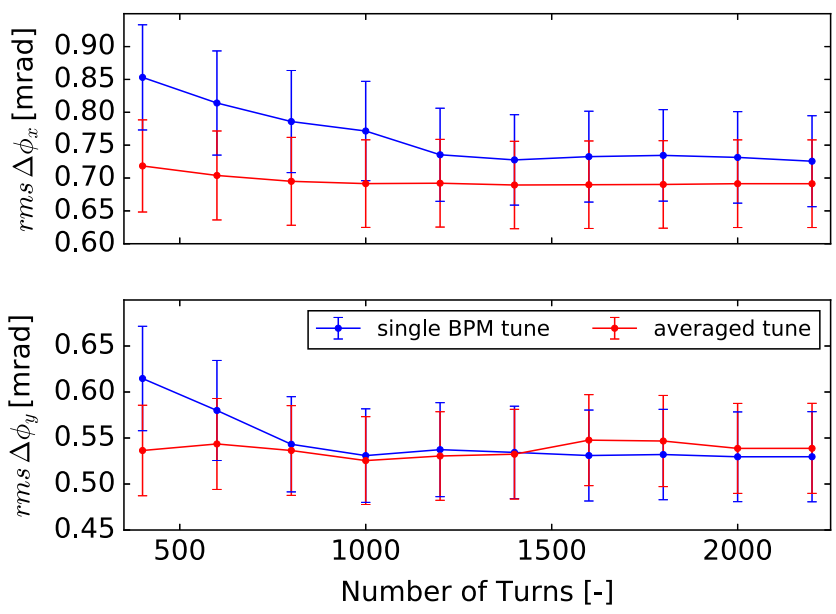

FIG. 2. Simulated rms phase-advance error computed from single-particle simulations of the ESRF storage ring lattice as a function of the number of turns used for the analysis in two cases where the betatron tune is calculated from a single BPMs or averaged over the set of all BPMs (realistic noise has been added to BPM TbT data). The initial displacement of $0.55 \mathrm{~mm}$ (horizontal) and $0.15 \mathrm{~mm}$ (vertical) at $\beta_{x}=38 \mathrm{~m}$ and $\beta_{y}=2.9 \mathrm{~m}$ (initial actions: $J_{x 0}=4.0 \times 10^{-9} \mathrm{~m}$ and $\left.J_{y 0}=3.9 \times 10^{-9} \mathrm{~m}\right)$.
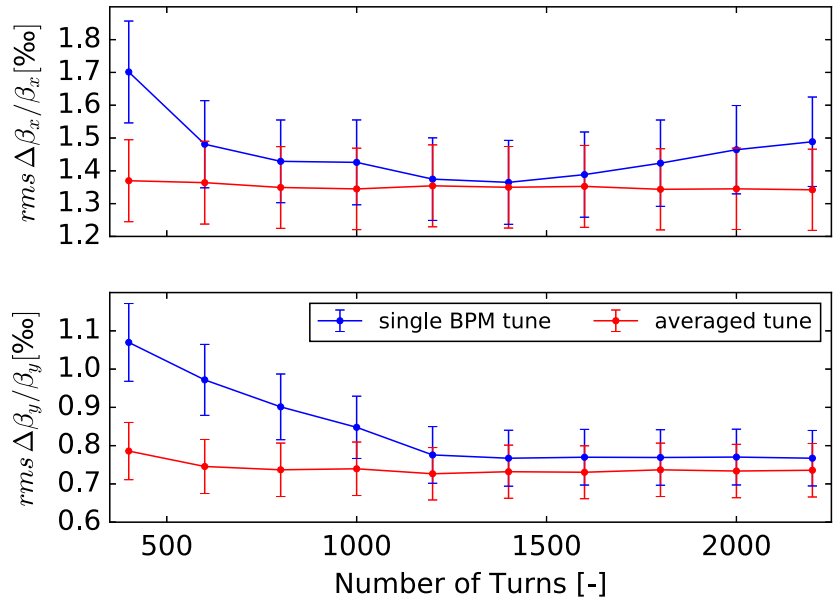

FIG. 3. Simulated rms of artificial $\beta$-beating computed by N-BPM from the same single-particle simulations of Fig. 2.

in the measurement of the horizontal $\beta$-functions an initial displacement below $0.7 \mathrm{~mm}$ (always at $\beta_{x}=38 \mathrm{~m}$ ) is needed, as shown in Fig. 5. The requirement is more relaxed in the vertical plane, for which an initial displacement below $0.17 \mathrm{~mm}$ at $\beta_{y}=2.9 \mathrm{~m}$, which is equivalent to $0.62 \mathrm{~mm}$ at $\beta_{y}=38 \mathrm{~m}$ in the horizontal plane, decreases the inaccuracy to $1 \%$. It is factor 2 lower compared to the horizontal plane. As the beam dynamics becomes nonlinear both phase and amplitude of the tune line are perturbed [11]. This may in turn cause a significant disagreement between the two methods at large amplitudes, as seen in Fig. 5.

TbT data with the lowest amplitude of the oscillation $(0.7 \mathrm{~mm})$ were chosen for the analysis since in this case the linear optics measurements are the least perturbed by amplitude detuning and nonlinearities. Moreover, the natural transverse damping induced by synchrotron radiation has been used to further decrease the systematic effect of
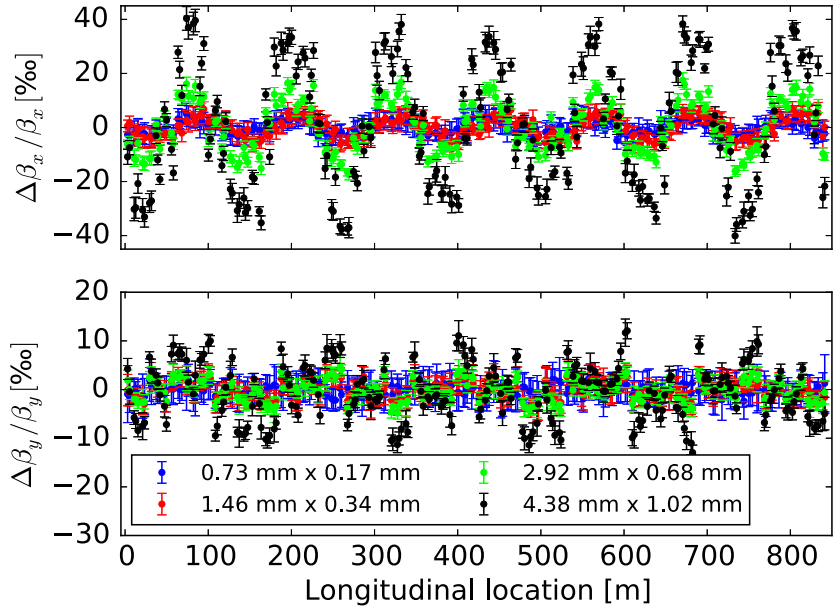

FIG. 4. Simulated artificial $\beta$-beating computed by N-BPM from single-particle simulations of the ESRF storage ring lattice at different initial displacements $\left(\beta_{x}=38 \mathrm{~m}\right.$ and $\left.\beta_{y}=2.9 \mathrm{~m}\right)$ corresponding to actions from $\sim 5 \times 10^{-9} \mathrm{~m}$ to $\sim 2.5 \times 10^{-7} \mathrm{~m}$. 

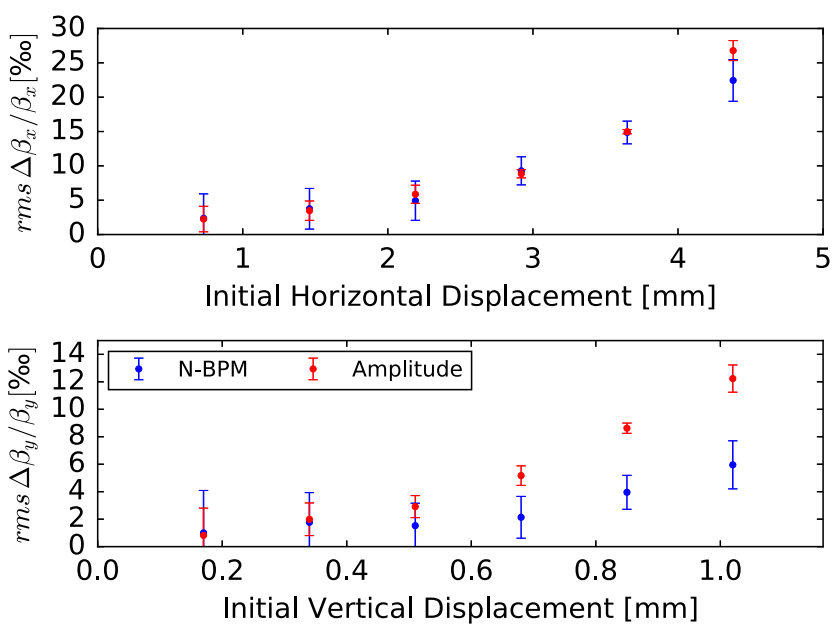

FIG. 5. Simulated rms of artificial $\beta$-beating computed by N-BPM and Amplitude methods from the same single-particle simulations with different diagonal initial displacement as in Fig. 4. The error bars refer to the mean (single-BPM) error bars averaged over all BPMs.

sextupoles, in our case by analyzing only a batch of data 200 turns after the kick (for larger kicks longer delay may be needed). Such a shift ensures a maximal transverse oscillation only slightly above $0.5 \mathrm{~mm}$ (horizontally, still at $\beta_{x}=38 \mathrm{~m}$ ) and $0.15 \mathrm{~mm}$ (vertically, $\beta_{y}=2.9 \mathrm{~m}$ ). After the raw data are SVD-cleaned, harmonic analysis is performed using an FFT with frequency interpolation by Jacobsen's method with bias correction [23]. The analyses and errors specific to the TbT methods are described in the following subsections.

\section{A. N-BPM method}

The $\beta$-function evaluated from the N-BPM method [13] is a weighted average of $\beta$-functions obtained from various combinations of BPM triplets, using model transfer matrices in between them. Therefore, uncertainties in the model influence the measurement of $\beta$-functions. Ten thousand lattices were simulated, to estimate the resulting systematic error on the $\beta$-function calculated from phase advances between a given combination of BPMs. To account for the effects of various sources of error, the estimated misalignments and uncertainties of magnetic properties were added to simulated lattices. The estimated uncertainties were tailored down in order to realistically reproduce the measured rms $\beta$-beating. The corresponding estimates of lattice uncertainties are shown in Table I.

The N-BPM method is influenced by phase advance measurement errors, therefore it is sensitive to errors in the synchronization among the BPMs. A phase advance variation has been observed both in simulation and in the measurement, when analysing the TbT data delayed even by a single turn. The variation resulted in rms $\beta$-beating up to $10 \%$ (with a delay of 3 or 4 turns as shown in Fig. 6). This was found to be an effect of the
TABLE I. Estimated Gaussian uncertainties of the ESRF lattice using the ORM inferred model.

\begin{tabular}{lr}
\hline \hline Uncertainty & $\sigma_{\text {ORM model }}$ \\
\hline Longitudinal quadrupole misalignment & $0.2 \mathrm{~mm}$ \\
Longitudinal BPMs misalignment & $0.2 \mathrm{~mm}$ \\
Transverse sextupole misalignment & $10 \mu \mathrm{m}$ \\
Quadrupole gradient & $0.1 \%$ o \\
BPM resolution (horizontal plane) & $9.6 \mu \mathrm{m}$ \\
BPM resolution (vertical plane) & $8.8 \mu \mathrm{m}$ \\
\hline \hline
\end{tabular}

transverse damping. In order to refine the phase advance measurement we implemented the phase correction given in [18] for a signal approximated by

$$
x_{k}=A e^{-\alpha k} \cos (\nu k+\phi+\epsilon) \text {, }
$$

where $k, A$, and $\alpha$ are the turn number, the amplitude and the damping coefficient. $\nu, \phi$, and $\epsilon$ are the betatron tune, the phase of the tune spectral line and the correction to the phase (all in radians), that is given by

$$
\epsilon=\frac{e_{1}-e_{2}}{e_{3}-e_{4}}
$$

where

$$
\begin{aligned}
& e_{1}=\frac{1}{2} \sum_{k} A e^{-2 \alpha k} \sin 2(\nu k+\phi) \\
& e_{2}=\sum_{k} x_{k} e^{-\alpha k} \sin (\nu k+\phi) \\
& e_{3}=\sum_{k} x_{k} e^{-\alpha k} \cos (\nu k+\phi) \\
& e_{4}=\sum_{k} A e^{-2 \alpha k} \cos 2(\nu k+\phi) .
\end{aligned}
$$
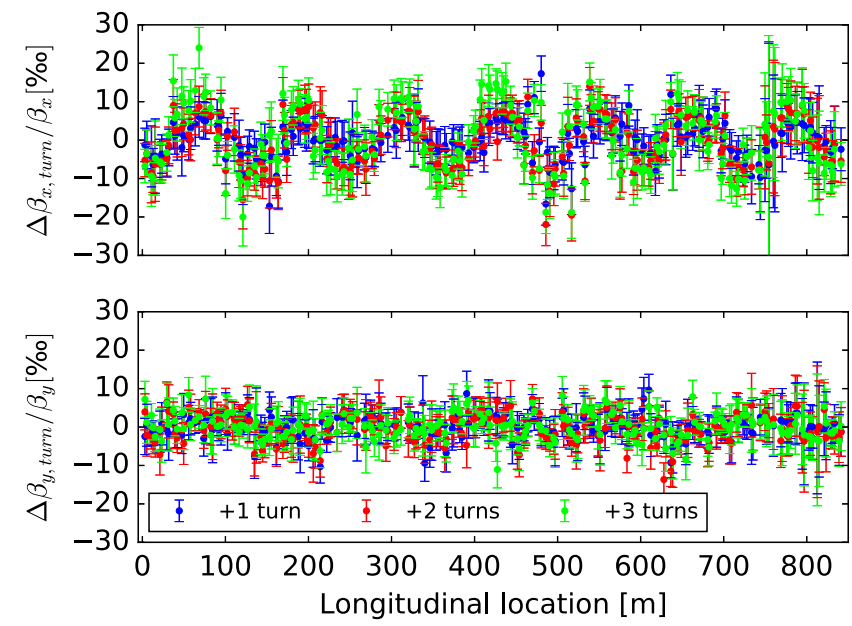

FIG. 6. Measured $\beta$-beating when analyzing the TbT data delayed by different number of turns. 


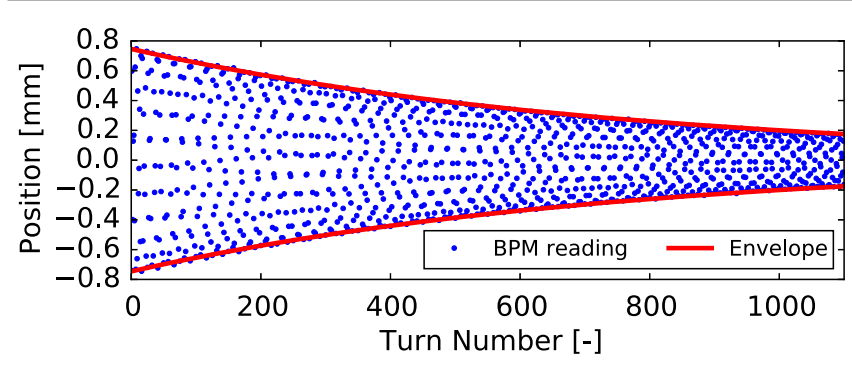

FIG. 7. TbT BPM position readings with estimated exponential envelope.

A robust estimator of the amplitude and damping coefficient has been developed and a sample of TbT data with its estimated exponential envelope is shown in Fig. 7. The correction gives stable results only if the average orbit is subtracted from the data. This improved the robustness of phase advance measurement, resulting in rms $\beta$-beating decreased to at most $0.4 \%$, typically even $0.2 \%$.

In order to account for the systematic error in the evaluation of the $\beta$-beating from nonlinear terms polluting the tune spectral line, single-particle simulations were run with initial amplitudes equivalent to those of the measurements. For each amplitude, the resulting artificial $\beta$-beating was quantified, as in Fig. 5, and its rms value was included in the systematic error associated to the analysis of experimental data.

\section{B. Amplitude method}

The betatron motion at a given BPM is described by Eq. (1). The undamped amplitude of the tune spectral line is

$$
A=C \sqrt{2 \beta J}
$$

where $J$ is the action (invariant along the ring), while $C$ and $\beta$ are the $\mathrm{BPM}$ gain $(\simeq 1)$ and $\beta$-function, respectively. All are entangled, as only the product $C \sqrt{\beta J}$ is observable. By assuming BPM gain is the same for all monitors, a way to extract $C \sqrt{J}$ (and hence the $\beta$-function at the BPMs) is to impose that model and measured average $\beta$-functions be equal, i.e. $K \cdot C^{2}\left\langle\beta^{(\text {meas })} J\right\rangle=\left\langle\beta^{(\bmod )}\right\rangle$, where $K$ is a global scaling factor. This approach adds in turn two systematic effect, because the average $\beta$-function increases with the rms $\beta$-beating $[11,24]$ and $C$ changes between BPMs. The BPM calibration errors can be indirectly estimated by comparing the $\beta$-functions obtained from N-BPM and Amplitude methods, the former not being affected by these errors.

The dependence of average $\beta$-beating on $\mathrm{rms} \beta$-beating of perturbed lattices with respect to the unperturbed model has been simulated. This turns out to be quadratic (in agreement with [24]), as shown in Fig. 8. The measured $\beta$-functions are corrected for this effect of rms $\beta$-beating.
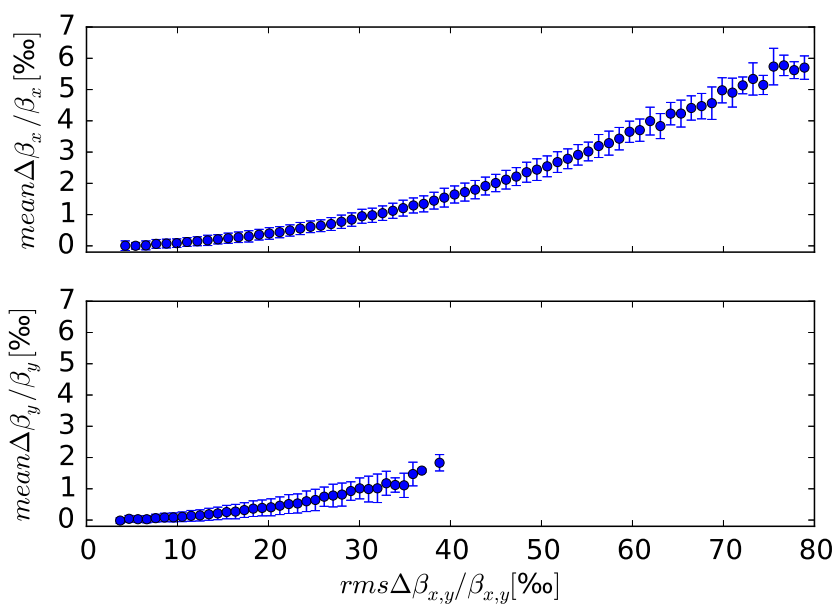

FIG. 8. Average $\beta$-beating vs rms $\beta$-beating (binned) of perturbed simulated lattices with respect to the unperturbed model of the ESRF storage ring (simulations).

The uncertainty in the simulated average $\beta$-beating (for a given rms $\beta$-beating) was included in the experimental systematic error, together with the artificial $\beta$-function accuracy mentioned above. The error in the analysis of experimental data consists of these systematic errors and statistical errors of $\beta$-function measurement.

\section{ORM method}

To the best of our knowledge, no theoretical study on the accuracy of the ORM method has been published so far. In order to evaluate a systematic error for the ESRF storage ring, numerical simulations have been carried out. Ten different sets of linear lattice errors generating the measured rms $\beta$-beating have been created. The ORM analysis has been simulated on those sets and the fitted models have been used to compute ten sets of $\beta$-functions at the BPMs. These have been compared to the expected values, yielding an rms uncertainty of about 3\%o. The inclusion of the BPM resolution at $10 \mathrm{~nm}$ level did not deteriorate that accuracy. In order to estimate the statistical error, we carried out 5 consecutive ORM measurements which resulted in a rms uncertainty of about $5 \%$ in the horizontal plane and about $2 \%$ in the vertical plane. This gives the measured $\beta$-function precision of $6 \%$ in horizontal plane and $4 \%$ o in vertical plane.

\section{COMPARISON OF RESULTS}

In order to measure the $\beta$-functions most precisely, the batch of TbT data 200 turns after the kick with the lowest amplitude was analyzed. In this case the linear optics is the least disturbed by amplitude detuning and non-linearities. The average relative precision of $\beta$-functions obtained by different methods is shown in Table II. The N-BPM precision is mainly consisting of the statistical error, and 
TABLE II. Relative precision of $\beta$-functions obtained by different methods.

\begin{tabular}{lcc}
\hline \hline Mean relative precision of $\beta \mathrm{s}$ & $x[\% \circ]$ & $y[\% \circ]$ \\
\hline N-BPM & 4 & 4 \\
Amplitude & 15 & 12 \\
ORM & 6 & 4 \\
\hline \hline
\end{tabular}

TABLE III. Measured $\beta$-beating between different methods.

\begin{tabular}{lcc}
\hline \hline Rms $\beta$-beating & $x[\%$ o & $y[\%$ ] \\
\hline N-BPM vs Amplitude & 17 & 12 \\
Amplitude vs ORM model & 20 & 13 \\
N-BPM vs ORM model & 11 & 9 \\
\hline \hline
\end{tabular}

remains at the level of $4 \%$ oven if lattice uncertainties are tripled. The precision of the Amplitude method is dominated by BPM gain errors, the contribution of all other sources of error together are less than 5\%o.

The measured $\beta$-beating is shown in Table III and Fig. 9. A drift of the closed orbit of about $15 \mu \mathrm{m} \mathrm{rms}$ was observed between ORM and TbT measurements: simulations suggest that about $5 \%$ of the difference in the $\beta$-beating between ORM and $\mathrm{TbT}$ techniques can be attributed to this orbit drift.

Phase advances between the neighboring BPMs (a more robust observable) were measured from $\mathrm{TbT}$ data and compared to those obtained from the ORM model. The difference is shown in Figure 10. The agreement is generally better in horizontal plane. Error bars shown in Fig. 10 are from the TbT measurement only, as there is no estimate of phase advance error inferred from the ORM method.

At a longitudinal location of around $500 \mathrm{~m}$, there is a local insertion optics. A larger than the average discrepancy is visible along that region both in the horizontal $\beta$-beating from the Amplitude method (Fig. 9) and in the phase advance beating between TbT measurement and ORM model (Fig. 10).This suggests the existence of local errors not included in the ORM model, in which the phase advances are not fit. The failure of the ORM method to reproduce the lattice errors, has been already observed in [25].

Finally, we compare the normalized dispersion $D / \sqrt{\beta}$, where $D$ stands for dispersion. Normalized dispersion was measured from closed orbit using ORM and from TbT data, their difference shown in Fig. 11. Differences between the two measurements are well within the error bars.

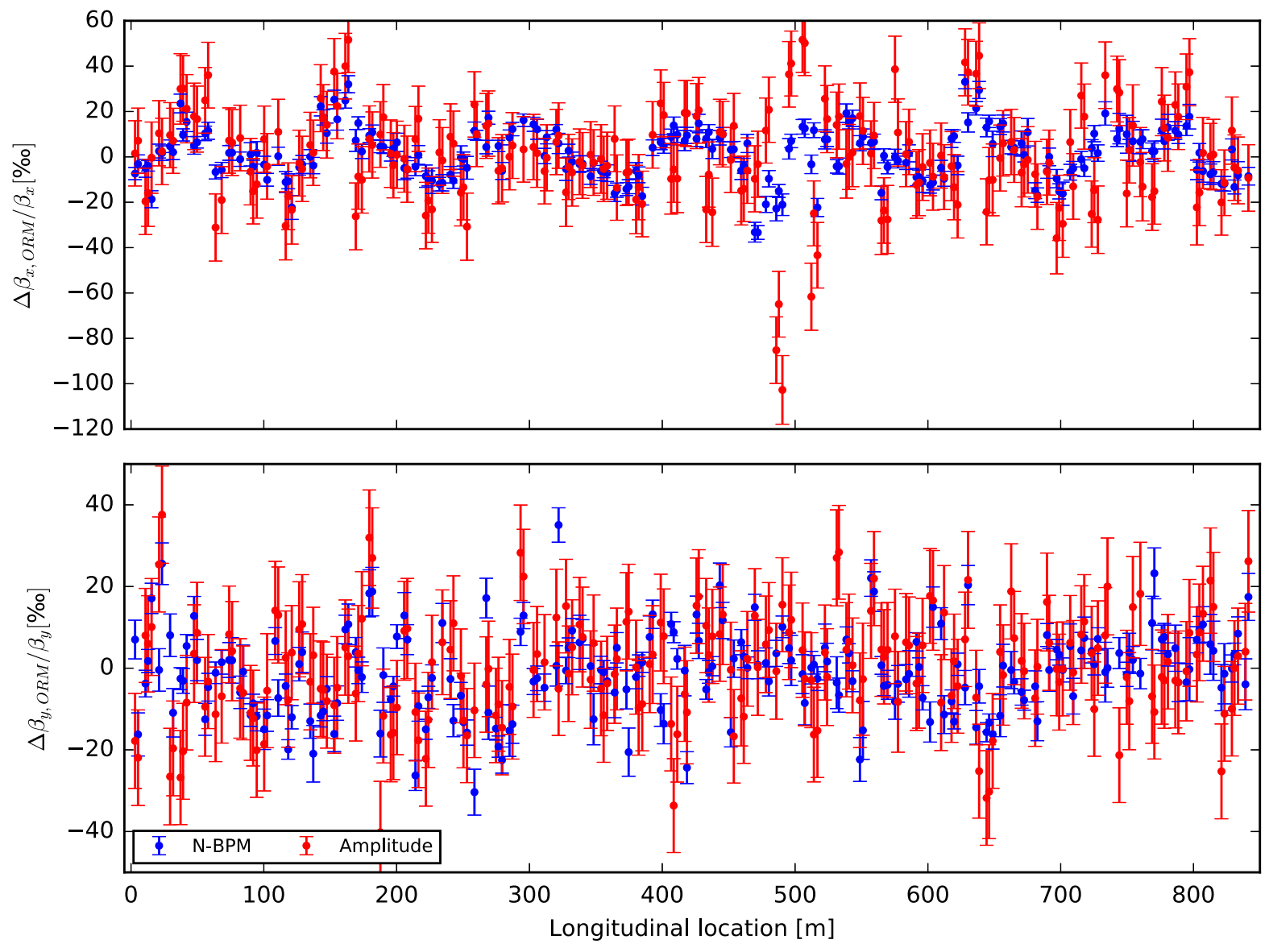

FIG. 9. Measured $\beta$-beating with respect to the ORM model. 

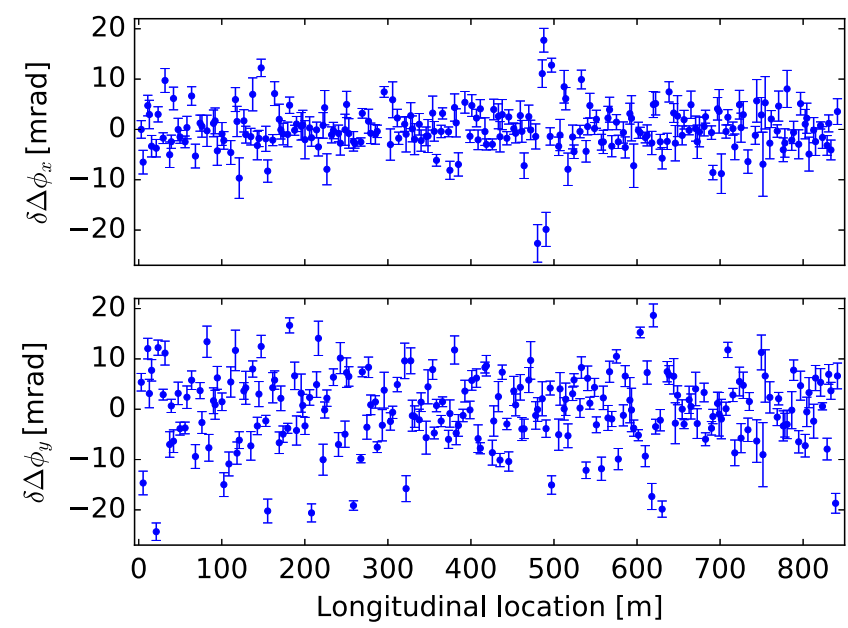

FIG. 10. Measured phase advance beating between neighboring BPMs with respect to the ORM model.
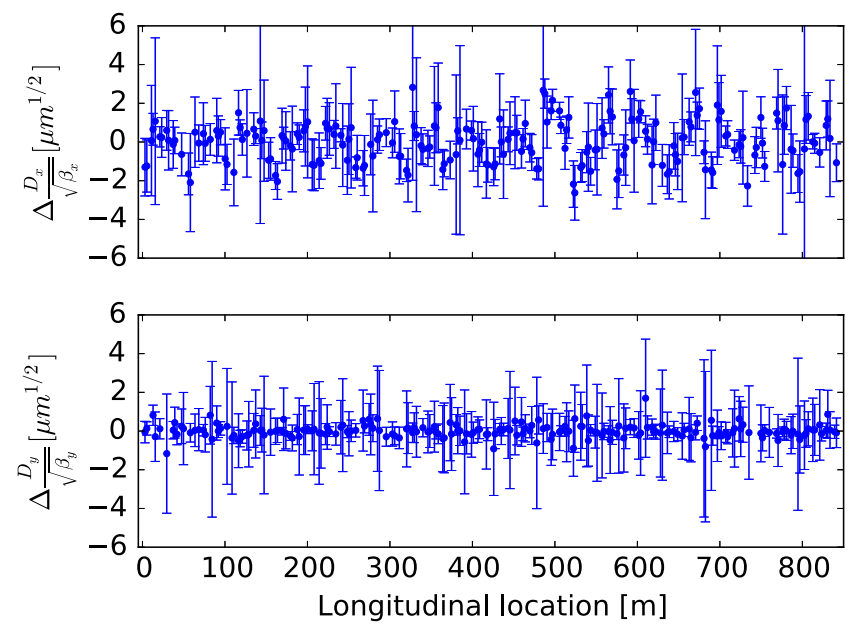

FIG. 11. Difference between measured normalized dispersion obtained from $\mathrm{TbT}$ data and from ORM.

\section{CONCLUSIONS AND OUTLOOK}

Three optics measurement methods were used and their results compared for the ESRF storage ring. A precision of $4 \%$ for evaluation of $\beta$-functions was obtained in both planes by the N-BPM method, as well as in the vertical plane from the ORM method. The horizontal plane from the ORM is slightly worse, reaching a precision of $6 \%$. The $\beta$-beating measured by N-BPM method and ORM are agree to the $10 \%$ o level, which gives an experimental upper limit estimate of the accuracy of the methods. Nevertheless, there is the issue of the time between the two measurements and possible orbit drifts may account for 5\%o. The agreement between the Amplitude method and the other two techniques is similar in the vertical plane, but factor 2 worse in the horizontal plane (up to $20 \%$ ).

Similar measurements were performed in the ALBA storage ring [9], which has the same BPM hardware and software. The BPM measurement noise at ALBA is about
$7 \mu \mathrm{m}$ [26], most probably due to lower level of mechanical vibrations making the beam more stable. The N-BPM method reached $8 \%$ o precision in the horizontal plane and $6 \%$ in the vertical plane [26] as defined in the Introduction. By counteracting the effect of oscillation damping and by using smaller kick amplitudes, as it is done here, most probably ALBA measurement precisions could be further improved.

Nevertheless, the $4 \%$ precision of the N-BPM method is expected to be limited to optics with low decoherence only, while the ORM method performance is less affected for optics with larger chromaticity and amplitude-dependent detuning. The quick decoherence of TbT BPM data can be overcome by replacing pulsed magnets (kickers) as source of excitation with AC-Dipoles [27], or shakers [28]. The beam is excited every turn (undergoing the forced oscillation), this way arbitrary transverse oscillation amplitudes are maintained for arbitrary number of turns independently of the optics. The induced forced oscillation would allow for thousands of exploitable turns at sufficiently low amplitude. The AC-Dipole has a variety of applications [22,29-32], however a detailed systematic study on the interplay between such excitation, radiation damping, and diffusion is still missing. The applicability of such device in the realm of lepton machines still needs to be assessed.

\section{ACKNOWLEDGMENTS}

The authors would like thank to the ESRF operators for the preparation of beam, to A. Langner who wrote the N-BPM method analysis software and to L. Farvacque who developed the data acquisition software.

[1] R. Tomás, M. Aiba, A. Franchi, and U. Iriso, Review of linear optics measurement and correction for charged particle accelerators, Phys. Rev. Accel. Beams 20, 054801 (2017).

[2] R. Tomás, M. Bai, R. Calaga, W. Fischer, A. Franchi, and G. Rumolo, Measurement of global and local resonance terms, Phys. Rev. ST Accel. Beams 8, 024001 (2005).

[3] R. Tomás, O. Brüning, M. Giovannozzi, P. Hagen, M. Lamont, F. Schmidt, G. Vanbavinckhove, M. Aiba, R. Calaga, and R. Miyamoto, CERN Large Hadron Collider optics model, measurements and corrections, Phys. Rev. ST Accel. Beams 13, 121004 (2010).

[4] A. Franchi, L. Farvacque, F. Ewald, G. LeBec, and K. B. Scheidt, First simultaneous measurement of sextupolar and octupolar resonance driving terms in a circular accelerator from turn-by-turn beam position monitor data, Phys. Rev. ST Accel. Beams 17, 074001 (2014).

[5] A. Langner et al., Developments of the segment-bysegment technique for optics corrections in the LHC, in Proceedings of IPAC'15, Richmond, USA, 2015 (JACoW, Geneva, Switzerland, 2015), paper MOPJE054. 
[6] X. Yang and X. Huang, Simultanous linear optics and coupling correction for storage rings with turn-by-turn beam position monitor data, arXiv:1511.02450v1.

[7] M. Aiba, M. Böge, J. Chrin, N. Milas, T. Schilcher, and A. Streun, Comparison of linear optics measurement and correction methods at the Swiss Light Source, Phys. Rev. ST Accel. Beams 16, 012802 (2013).

[8] G. Wang et al., Experimental crosscheck of algorithms for magnet lattice correction, in Proceedings of IPAC'16, Busan, Korea, 2016 (JACoW, Geneva, Switzerland, 2016), paper THPMR008.

[9] A. Langner, G. Benedetti, M. Carlà, U. Iriso, Z. Martí, J. Coello de Portugal, and R. Tomás, Utilizing the $N$ beam position monitor method for turn-by-turn optics measurements, Phys. Rev. Accel. Beams 19, 092803 (2016).

[10] Y. Jiao and Z. Duan, Statistical analysis of the limitation of half integer resonances on the available momentum acceptance of the High Energy Photon Source, Nucl. Instrum. Methods Phys. Res., Sect. A 841, 97 (2017).

[11] A. Franchi, Error analysis of linear optics measurements via turn-by-turn beam position data in circular accelerators, arXiv:1603.00281v2.

[12] L. Malina et al., Comparison of optics measurements methods in ESRF, in Proceedings of IPAC'16, Busan, Korea, 2016 (JACoW, Geneva, Switzerland, 2016), paper THPMB045.

[13] A. Langner and R. Tomás, Optics measurement algorithms and error analysis for the proton energy frontier, Phys. Rev. ST Accel. Beams 18, 031002 (2015).

[14] J. Borer et al., Harmonic analysis of coherent bunch oscillations in LEP, in Proceedings of EPAC'92, Berlin, Germany, 1992 (ASF, Berlin, Germany, 1992), p. 1082.

[15] A. Garcia-Tabares Valdivieso et al., Optics-measurementbased BPM calibration, in Proceedings of IPAC'16, Busan, Korea, 2016 (JACoW, Geneva, Switzerland, 2016), paper THPMB041.

[16] W. J. Corbett et al., A fast model-calibration procedure for storage rings, in Proceedings of the 15th Particle Accelerator Conference, PAC-1993, Washington, DC, 1993 (IEEE, New York, 1993), p. 108.

[17] B. K. Scheidt and F. Epaud, Installation and commissioning of a complete upgrade of the BPM system for the ESRF storage ring, in Proceedings of DIPAC09, Basel, Switzerland, 2009, (JACoW, Basel, Switzerland, 2009), p 50.

[18] W. Guo et al., A lattice correction approach through betatron phase advance, in Proceedings of IPAC'16, Busan, Korea, 2016 (JACoW, Geneva, Switzerland, 2016), paper MOOCB02.
[19] J. Irwin, C. X. Wang, Y. T. Yan, K. L. F. Bane, Y. Cai, F.-J. Decker, M. G. Minty, G. V. Stupakov, and F. Zimmermann, Model-Independent Beam Dynamics Analysis, Phys. Rev. Lett. 82, 1684 (1999).

[20] R. Calaga and R. Tomás, Statistical analysis of RHIC beam position monitors performance, Phys. Rev. ST Accel. Beams 7, 042801 (2004).

[21] X. Huang, S. Y. Lee, E. Prebys, and R. Tomlin, Application of independent component analysis to Fermilab Booster, Phys. Rev. ST Accel. Beams 8, 064001 (2005).

[22] N. Biancacci and R. Tomás, Using AC dipoles to localize sources of beam coupling impedance, Phys. Rev. Accel. Beams 19, 054001 (2016).

[23] Ç. Candan, A method for fine resolution frequency estimation from three DFT samples, IEEE Signal Proccessing Letters 18, 351 (2011).

[24] R. Calaga, R. Tomás, and F. Zimmermann, BPM calibration independent LHC optics correction, in Proceedings of the 22nd Particle Accelerator Conference, PAC-2007, Albuquerque, NM (IEEE, New York, 2007), p. 3693.

[25] M. Aiba and M. Böge, Local orbit response matrix measurement at SLS, in Proceedings of IPAC'15, Richmond, USA, May 2015 (JACoW, Geneva, Switzerland, 2015), paper TUPJE044.

[26] A. Langner (private communication).

[27] M. Bai, S. Y. Lee, J. W. Glenn, H. Huang, L. Ratner, T. Roser, M. J. Syphers, and W. van Asselt, Experimental test of coherent betatron resonance excitations, Phys. Rev. E 56, 6002 (1997).

[28] D. Sagan, R. Meller, R. Littauer, and D. Rubin, Betatron phase and coupling measurements at the Cornell Electron/ Positron Storage Ring, Phys. Rev. ST Accel. Beams 3, 092801 (2000).

[29] R. Tomás et al., Record low beta beating in the LHC, Phys. Rev. ST Accel. Beams 15, 091001 (2012).

[30] S. White, E. Maclean, and R. Tomás, Direct amplitude detuning measurement with AC dipole, Phys. Rev. ST Accel. Beams 16, 071002 (2013).

[31] T. H. B. Persson, Y. Inntjore Levinsen, R. Tomas, and E. H. Maclean, Chromatic coupling correction in the Large Hadron Collider, Phys. Rev. ST Accel. Beams 16, 081003 (2013).

[32] S. Mönig et al., Short term dynamic aperture with AC-Dipole DA, in Proceedings of IPAC'16, Busan, Korea, 2016 (JACoW, Geneva, Switzerland, 2016), paper THPMR044. 